PAPER • OPEN ACCESS

Influence of minor additions on Icosahedral Short-Range Order (ISRO) and its consequences on nucleation and solidification growth kinetics in fcc alloys

To cite this article: J. Zollinger and M. Rappaz 2019 IOP Conf. Ser.: Mater. Sci. Eng. 529012045

View the article online for updates and enhancements. 


\title{
Influence of minor additions on Icosahedral Short-Range Order (ISRO) and its consequences on nucleation and solidification growth kinetics in fec alloys
}

\author{
J. Zollinger ${ }^{1}$, M. Rappaz $^{2}$ \\ ${ }^{1}$ Institut Jean Lamour, Department of Metallurgy and \\ Materials Science \& Engineering, Université de Lorraine, Campus ARTEM, \\ Allée André Guinier, F-54011 Nancy, France \\ ${ }^{2}$ Institute of Materials, École Polytechnique Fédérale de Lausanne, \\ Station 12, CH-1015 Lausanne, Switzerland \\ julien.zollinger@univ-lorraine.fr
}

\begin{abstract}
The present contribution reviews the recent progress related to the influence of Icosahedral Short-Range Order (ISRO) and icosahedral Quasicrystals (i-QC) formation on the solidification of fcc alloys through minor solute element additions. From intensive crystallographic analysis of multi-twinned regions in as-cast Al-based and Au-based fec alloys, Kurtuldu et al. have shown recently that a so-called "iQC-mediated" nucleation mechanism occurs when a few hundred ppm of $\mathrm{Cr}$ and Ir, respectively, are added to the melt [1] [2]. Similarly, it appears that the growth directions of dendrites in Al-Zn:Cr is also influenced by ISRO in the liquid, thus showing an attachment kinetics effect [3]. In a recent contribution, we have shown that iQC-mediated nucleation also occurs in pink gold alloys with Ir-additions, but two additional phenomena at high solidification speed [4]: (i) a spinodal-type decomposition of the liquid, leading to the formation of twinned $\mathrm{Cu}$ precipitates in addition to multi-twinned $\mathrm{Au}$ rich grains; (ii) a change of the microstructure of the Au-rich grains, from $\langle 100\rangle$ dendrites to $\langle 111\rangle$ textured cells in the columnar zone.
\end{abstract}

\section{Introduction}

Icosahedral Short Range Order (ISRO) in the liquid phase, conjectured by Frank in 1952 [5], was thought to be responsible for the large undercooling measured in metallic alloys. This preferred local arrangement of atoms in the liquid, due to local covalent bonding, was confirmed to occur by atomistic calculations and by small angle scattering [6]. ISRO is also key in understanding the formation of QuasiCrystals (QC) discovered by Shechtman et al. in Al-Mn alloys [7], and later in many other alloys [8]. Through several recent papers, Kurtuldu and Rappaz have shown that ISRO can also promote the formation of fine equiaxed fcc grains through a mechanism which they referred to as "iQC-mediated nucleation", the " $i$ " standing for icosahedral [1][2][9]. This mechanism is summarized in Figure 1 for $\mathrm{Al}-\mathrm{Zn}: \mathrm{Cr}$. In the liquid state (a), $\mathrm{Al}$ and $\mathrm{Zn}$ atoms tend to be localized in ISRO configuration around smaller $\mathrm{Cr}$ atoms. As the liquidus of the $\mathrm{Al}_{7} \mathrm{Cr}$ phase is first crossed during cooling, even at very low $\mathrm{Cr}$ composition, $\mathrm{iQC}$ tend to form easily (b), since the interfacial energy between the liquid and $\mathrm{QQC}$ is at least an order of magnitude lower than that with the fcc phase [8]. As the temperature is further lowered, 
IOP Conf. Series: Materials Science and Engineering 529 (2019) 012045 doi:10.1088/1757-899X/529/1/012045

the liquid surrounding the $\mathrm{iQC}$ is depleted in $\mathrm{Cr}$ atoms, thus creating a driving force to form the fcc phase (c), with the following heteroepitaxy relationships with the iQC template:

\section{$\langle 111\rangle_{f c c} / / 3$-fold symmetry of $i Q C \quad$ and $\quad\langle 110\rangle_{f c c} \perp 2$-fold symmetry axis of iQC}

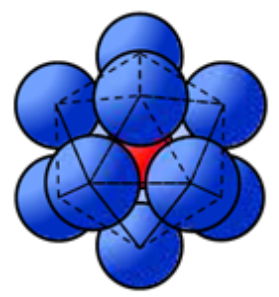

(a)

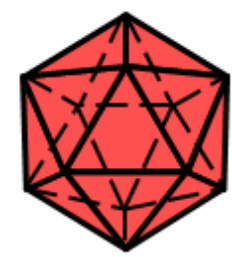

(b)

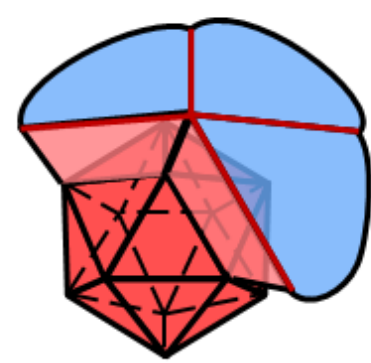

(c)

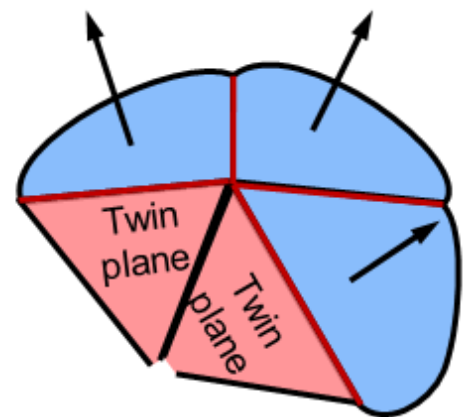

(d)

Figure 1: Summary of the iQC-mediated nucleation mechanism (from [2]).

As a result, grains forming on the facets of the iQC have Multiple Twin (MT) relationships among them, resulting in symmetry relationships similar to what was observed in the 1960's in small nanocrystals of gold [10]. During further solidification, the central Cr-rich iQC can even disappear since $\mathrm{Al}-\mathrm{Cr}$ is peritectic (d) [11]. As a matter of fact, Kurtuldu et al. discovered this mechanism from the excessive number of twinned grain boundaries in $\mathrm{Al}-20 \mathrm{wt} \% \mathrm{Zn}$ when $0.1 \mathrm{wt} \% \mathrm{Cr}$ is added. They also found MT relationships among several nearest-neighbor (nn) fcc grains, respecting the icosahedral symmetry of the iQC template. In some cases, they found the $\mathrm{nn}$ fcc grains to have symmetry relationships compatible with the so-called interlocked icosahedron (two icosahedra linked via pentagonal edges).

The same mechanism was then shown to operate in yellow gold $(\mathrm{Au}-12.5 \mathrm{wt} \% \mathrm{Ag}-12.5 \mathrm{wt} \% \mathrm{Cu})$ when minute amounts of Ir are added to the melt (from 0.001 to $0.02 \mathrm{wt} \%$ ), a technique used since many years in jewelry and watch industries for grain refining [12].

When ISRO occurs in the liquid, Kurtuldu et al. also showed that solute diffusion is slowed down since the mobility of these clusters is reduced compared to the situation when localized covalent bonds are not present [13]. If such is the case, ISRO should also impact the growth kinetics of the fcc phase. Already in his $\mathrm{PhD}$ thesis, Kurtuldu reported that the growth directions of dendrite trunks and arms are changed in $\mathrm{Al}-\mathrm{Zn}$ alloys when $\mathrm{Cr}$ is added, and become a function of the solidification speed [3].

Recently, we have shown that iQC-mediated nucleation occurs also in pink gold alloys (Au$20.5 \mathrm{wt} \% \mathrm{Cu}-4.5 \mathrm{wt} \% \mathrm{Ag}$ ) when $0.01 \mathrm{wt} \%$ Ir atoms are added to the melt [4]. While this mechanism could be expected at low solidification speed, since it is also used routinely in jewelry for grain refining, two additional phenomena were found at high solidification rate: (i) the formation of fine $\mathrm{Cu}$ precipitates, sometimes with twinning relationships, in addition to MT Au-rich grains; and (ii) a change of the internal morphology and texture of columnar grains. These last results are briefly summarized in the present contribution.

\section{Experimental method and analysis}

Pink gold alloys with and without 100 wt. ppm Ir additions, were supplied by PX Group. Low solidification rate (typically $-0.25 \mathrm{~K} / \mathrm{s}$ ) was achieved in a DSC calorimeter, while rapid cooling experiments were performed by letting a small liquid drop (about $4 \mathrm{~g}$ ) fall onto a water-cooled $\mathrm{Cu}$ 
IOP Conf. Series: Materials Science and Engineering 529 (2019) 012045 doi:10.1088/1757-899X/529/1/012045

substrate. In this case, the cooling rate is typically $-10^{4} \mathrm{~K} / \mathrm{s}$ near the copper chill and $-10^{2} \mathrm{~K} / \mathrm{s}$ near the top of the solidified drop. After cutting and polishing, the grain and microstructure were observed using optical microscopy, SEM and EBSD. Further experimental details are given in [4] and we report below only the results concerning rapidly cooled specimens, with and without Ir addition.

\section{Results and discussion}

Figure 2 summarizes the BSE analysis results of rapidly solidified pink gold droplets without (top) and with (bottom) Ir. In this figure, the copper chill is located on the left (i.e., gravity is from right to left) and the left figures have been taken in the part of the droplets which was in contact with the chill. The two figures on the right correspond to the equiaxed zone, in the upper part of the droplets. Small inserts have been added in each figure to emphasize better the internal microstructure of the grains. Au-rich grains appear with various shades of light grey in BSE, while $\mathrm{Cu}$-rich regions located either in between dendrite arms or as precipitates (see discussion below) are much darker.
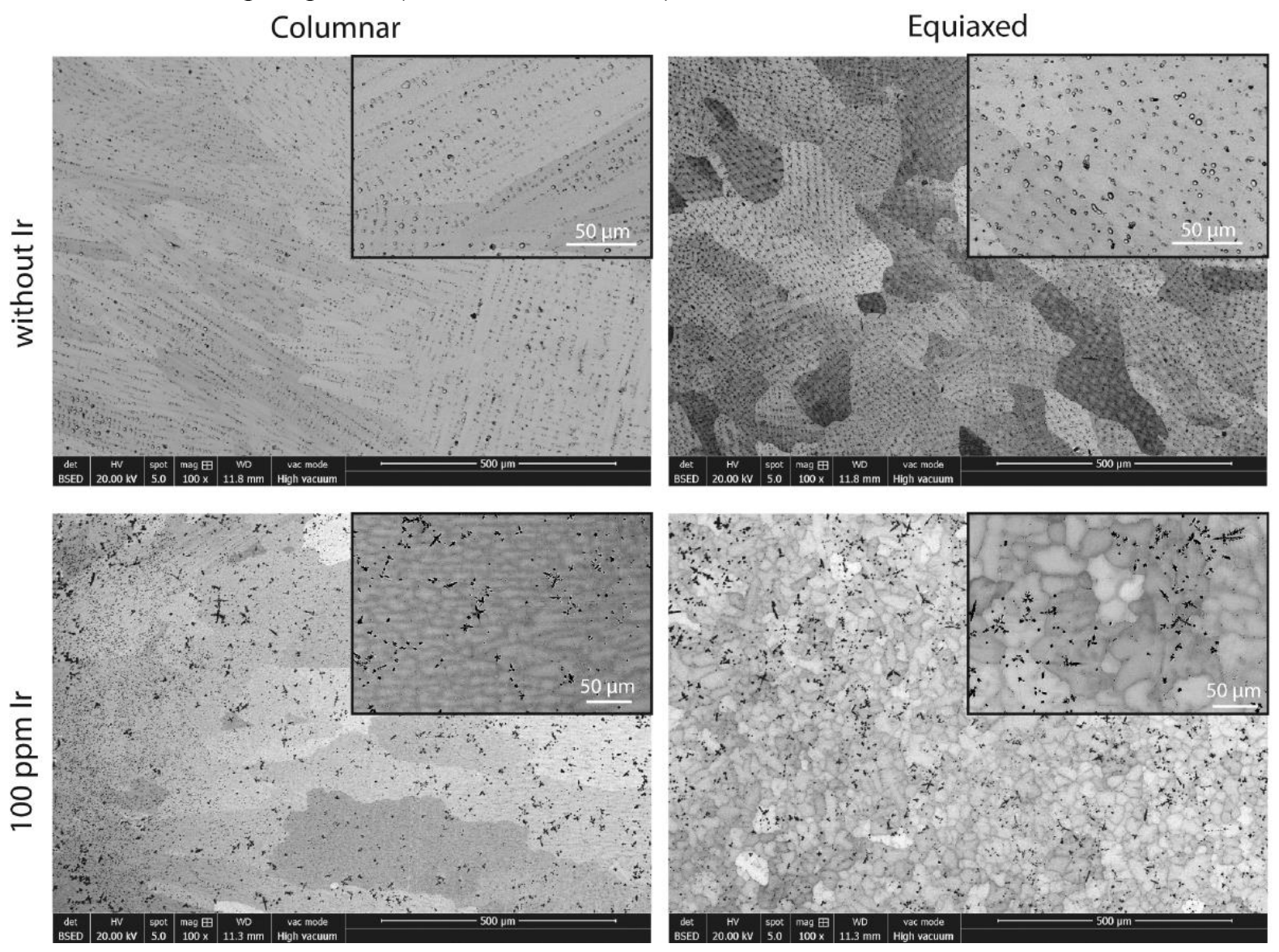

Figure 2: Backscattered electrons image showing the microstructure of Au-20.5wt.\%Cu$4.5 \mathrm{wt} . \% \mathrm{Ag}$ specimens rapidly solidified without (top) and with (bottom) $0.01 \mathrm{wt} \%$ Ir. The left and right figures correspond to the columnar and equiaxed zones, respectively, the copper chill being located on the left. For each figure, an enlarged view of the internal microstructure of the grains is shown in the small inserts.

The grain structure of the droplets was also analyzed by EBSD in order to determine the orientation of the grains and detect twin boundary relationships, i.e., rotation of $60 \mathrm{deg}$. around a common $\langle 111\rangle-$ axis, within an accuracy of $5 \mathrm{deg}$. as in [1,2]. Figure 3 (a) and (b) show false-color reconstruction of 
cross sections of rapidly solidified pink gold droplets. The part of the droplet in contact with the $\mathrm{Cu}$ plate is on the left and exhibits columnar grains, while the upper part of the droplet (slowest cooling rate) is on the right and exhibit equiaxed grains. Twinned grain boundaries are shown in white. Multiple twin relationships were also found between nn Au-rich equiaxed grains, as illustrated in Figure 3(c) for 4 grains with $0.01 \mathrm{wt} \%$ Ir addition. The corresponding $\langle 110\rangle$ pole figure shows their multiple twin relationships, with the common $\langle 110\rangle$ direction corresponding to the 5-fold symmetry axis of an icosahedron, the common $\{111\}$ planes being shown with arcs of circle.

(a)

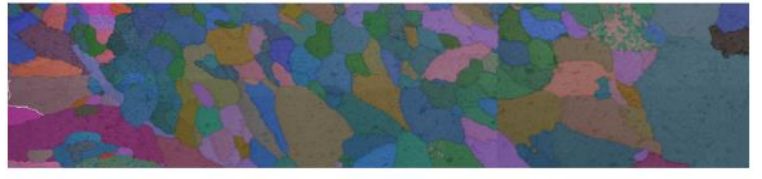

(b)

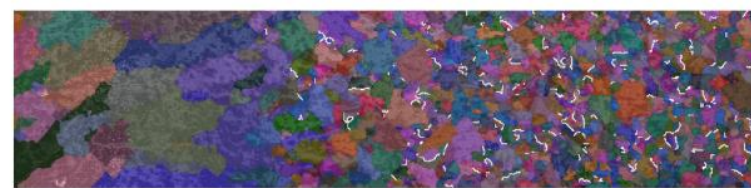

(c)
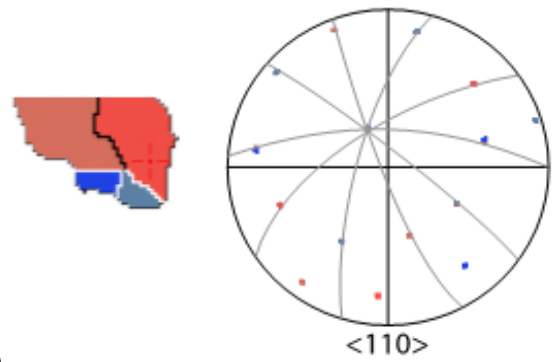

Figure 3: EBSD reconstructed false-color map of a section through a rapidly solidified pink gold droplet (a) without and (b) with $0.01 \mathrm{wt} \% \mathrm{Ir}$. Twin grain boundaries are shown in white. (c) An example of $4 \mathrm{nn}$ multiple twin pink gold grains present in (b) with corresponding $\langle 110\rangle$ pole figures. The common $\{111\}$ planes are shown with arc of circles.

These observations first show that minute additions of Ir induce similar i-QC and ISRO effects in pink gold alloys [2]:

- Iridium refines the grain structure of Au-rich equiaxed grains (compare Figure 2 right, top and bottom, or Figure 3(a) and (b)). This grain refinement is accompanied by a large percentage of twin grain boundaries $(7.6 \%)$, much larger than the percentage obtained for a population of randomly oriented grains $(0.2 \%)$. This percentage of grain boundaries is almost independent of the cooling rate, as observed in slowly cooled pink gold specimens with the same amount of iridium [4].

- Multiple twin or near-twin relationships between several nn Au-rich grains have been observed in the Ir-containing specimen. As illustrated in Figure 3(c) for 4 grains, they respect the 5-fold symmetry of the icosahedron with a common $\langle 110\rangle$ crystallographic axis.

- It might be surprising that the columnar zone of the specimen with Ir does not exhibit any twin, and so does that of the Ir-free specimen. However, this is expected even if nucleation at or on the copper chill surface is mediated by the formation of $\mathrm{iQC}$. Indeed, during growth, grain competition cannot favor two adjacent grains coming from the same iQC template and thus observed twins, if any, can only result from randomness. Surprisingly, while the equiaxed grains are refined with Ir addition, the columnar zone becomes more extended: it is about $1.2 \mathrm{~mm}$ long with $\mathrm{Ir}$ and only $0.5 \mathrm{~mm}$ without Ir. This seems to imply that the growth kinetics is also affected by Ir addition.

Indeed, with the high solidification rate associated with the falling droplet, several new phenomena have been observed showing that ISRO in the liquid also influences growth:

- First, the internal microstructure of the columnar grains with Ir is markedly different from that without Ir (compare Figure 2 left, top and bottom). Without Ir, the columnar grains are clearly dendritic and exhibit a $\langle 100\rangle$ texture, as commonly observed in most metallic fec alloys where competition of $\langle 100\rangle$ dendrite trunks occurs [14]. With Ir, the dendritic structure is replaced by cells growing in a direction more or less parallel to the thermal gradient direction (i.e., from left to right in Figure 2). Furthermore, these columnar grains exhibit a $\langle 111\rangle$ texture, thus showing an effect of 
ISRO in the liquid on the attachment kinetics during growth. To some extent, equiaxed grains exhibit a similar change, i.e., dendritic without Ir and globular-dendritic with Ir, but it is less evident. First, the grains are very small and the microstructure becomes naturally more globulardendritic. Second, the cooling rate is reduced in this part of the droplet which, associated with the reduced grain size, reduces the interface velocity.

- Finally, besides the MT Au-rich grains appearing in various light grey shades in Figure 2, one can observe that small black precipitates or even dendrites appear in BSE when Ir is added. EDX measurements have revealed that these precipitates are a $\mathrm{Cu}$-rich phase, i.e., composition higher than $95 \mathrm{wt} \%$ in $\mathrm{Cu}$. It is to be noted that this phase does not appear in DSC when the solidification speed is low. The size and morphology of these $\mathrm{Cu}$-rich particles/dendrites clearly indicate that they did not form by precipitation in the solid state and solidify even before the Au-rich MT grains, as shown in Figure 4(a). The EBSD analysis of these precipitates is shown in Figure 4(b-c). It appears that these equiaxed $\mathrm{Cu}$-rich dendrites most often exhibit twins and probably result from a similar nucleation mechanism induced by ISRO.

This nearly simultaneous formation of $\mathrm{Cu}$-rich precipitates/dendrites and $\mathrm{Au}$-rich grains, which only occurs at high solidification rate when $0.01 \mathrm{wt} \%$ Ir is added to the melt, seems to indicate that Ir induces a spinodal-type decomposition of the liquid via ISRO. The $\mathrm{Cu}$-Ir phase diagram is peritectic, with a peritectic composition around 5at\% [15], and thus the mechanism is similar to that prevailing in Al-Zn:Cr, i.e., depletion of $\mathrm{Ir}$ around $\mathrm{iQC}$ and driving force to form $\mathrm{Cu}$. Unfortunately, the Au-Ir phase diagram is not clearly established: a reference mentions a eutectic with a very low eutectic composition (50 at ppm) [16], and another a peritectic with a much larger peritectic composition $(0.5$ at $\%)$ [17].

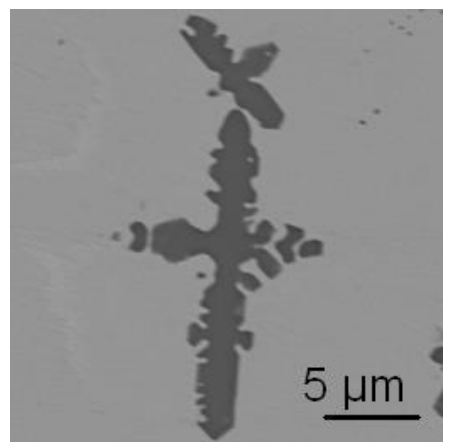

(a)

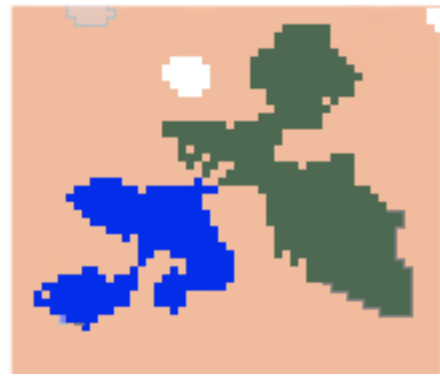

(b)
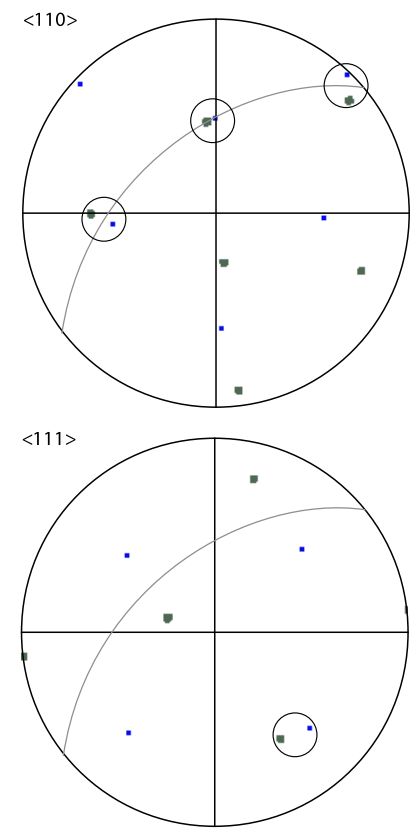

(c)

Figure 4: (a) Equiaxed copper dendrites observed 500 $\mu \mathrm{m}$ from the chill in the sample with $0.01 \mathrm{wt} \% \mathrm{Ir}$. The matrix is fcc-gold and corresponds to the columnar zone in Fig. 3(b). (b) False color EBSD map of similar copper dendrites, and (c) corresponding pole figures. 


\section{Conclusion}

Addition of small amounts of Ir in pink gold induces the same iQC-mediated nucleation of the fcc phase, similarly to what has been reported in $\mathrm{Al}-\mathrm{Zn}: \mathrm{Cr}$ and in yellow gold with Ir. First, the Au-rich grains are dramatically refined with a large percentage of twinned grain boundaries. Second, multiple twins relationships between Au-rich grains have been found: they reproduce the symmetry of the icosahedron template. These observations are not surprising and while MT boundaries have been the only phenomenon to be seen at low solidification speed, two new phenomena have been observed at high solidification rate. First, the internal microstructure of the grains is clearly different, especially for columnar grains for which a change of the texture from $\langle 100\rangle$ to $\langle 111\rangle$ is observed when Ir is added. This can be explained if one considers the attachment kinetics of ISRO clusters in the liquid on the growing fcc phase. Similar observations were made by Kurtuldu in directional solidification of Al-Zn:Cr dendrites when the solidification speed is increased. Secondly, it is shown that this mechanism, in undercooled melt, can lead to the nucleation of metastable phase, here copper-rich dendrites/precipitates that exhibit similar twin boundary relationships.

\section{References}

[1] G. Kurtuldu, P. Jarry, and M. Rappaz, 'Influence of Cr on the nucleation of primary Al and formation of twinned dendrites in Al-Zn-Cr alloys: Can icosahedral solid clusters play a role?', Acta Mater., vol. 61, no. 19, pp. 7098-7108, Nov. 2013.

[2] G. Kurtuldu, A. Sicco, and M. Rappaz, 'Icosahedral quasicrystal-enhanced nucleation of the fcc phase in liquid gold alloys', Acta Mater., vol. 70, pp. 240-248, May 2014.

[3] G. Kurtuldu, 'Influence of trace elements on the nucleation and solidification morphologies of fcc alloys and relationship with icosahedral quasicrystal formation', PhD thesis, EPFL, Lausanne, 2014.

[4] J. Zollinger, B. Rouat, S. K. Pillai, and M. Rappaz, 'Influence of Ir additions and icosahedral short range order (ISRO) on nucleation and growth kinetics in $\mathrm{Au}-20.5 \mathrm{wt} \% \mathrm{Cu}-4.5 \mathrm{wt} \% \mathrm{Ag}$ alloy', Metal. Mater. Trans. A, accepted publication, 2019.

[5] F. Frank, 'Supercooling of liquids', Proc. R. Soc. Lond. Ser. -Math. Phys. Sci., vol. 215, no. 1120, pp. 43-46, 1952.

[6] H.-R. Trebin, Quasicrystals: Structure and physical properties. John Wiley \& Sons, 2006.

[7] D. Shechtman, I. Blech, D. Gratias, and J. Cahn, 'Metallic phase with long-range orientational order and no translational symmetry', Phys. Rev. Lett., vol. 53, no. 20, pp. 1951-1953, 1984.

[8] K. F. Kelton, 'Quasicrystals: Structure and stability', Int. Mater. Rev., vol. 38, no. 3, pp. 105137, Jan. 1993.

[9] M. Rappaz and G. Kurtuldu, 'Thermodynamic aspects of homogeneous nucleation enhanced by icosahedral short range order in liquid fcc-type alloys', JOM, vol. 67, no. 8, pp. 1812-1820, Aug. 2015.

[10] S. Ino, 'Stability of multiply-twinned particles', J. Phys. Soc. Jpn., vol. 27, no. 4, pp. 941-953, Oct. 1969.

[11] G. Kurtuldu, P. Jessner, and M. Rappaz, 'Peritectic reaction on the Al-rich side of Al-Cr system', J. Alloys Compd., vol. 621, pp. 283-286, Feb. 2015.

[12] D. Ott and C. J. Raub, 'Grain size of gold and gold alloys', Gold Bull., vol. 14, no. 2, pp. 69-74, Jun. 1981.

[13] G. Kurtuldu, P. Jarry, and M. Rappaz, 'Influence of icosahedral short range order on diffusion in liquids: A study on Al-Zn-Cr alloys', Acta Mater., vol. 115, pp. 423-433, Aug. 2016.

[14] M. Rappaz and J. A. Dantzig, Solidification. EPFL Press, 2nd Edition, 2016.

[15] E.Rauband, E.Roeschel.Diekupfer-iridium-legierungen.Z.Metallkd.,60:142-144,1969.

[16] Au-Ir binary phase diagram. http://ipandat.computherm.com/Diagram/Details/7556, 2017.

[17] J.P. Nielsenand, J.J. Tuccillo. Grain size in cast gold alloys. Journal of Dental Research, 45(3):964-969,1966. 